\title{
High-speed Target Recognition Algorithm and Simulation
}

\author{
Shuai Duan \& Jiahao Deng \\ ${ }^{1}$ School of Mechatronical Engineering, Beijing Institute of Technology, Beijing, China; \\ ${ }^{2}$ Science and Technology on Electromechanical Dynamic Control Laboratory, Beijing, China
}

\begin{abstract}
The purpose of this paper is devoted to finding a method to recognize the high-speed target for missile in a certain distance. Based on the quasi-electrostatic field characteristics of the capacitance detector, this paper analyzed the output voltage of detection circuit from a capacitance detector. It can be found that, when a high-speed target getting close to the detector, the output voltage of detection circuit changes with certain rules. Our research applied a low-speed static test to obtain the static characteristic data of the target, proposed a high-speed target recognition algorithm and set threshold values based on starting voltage, second derivative and signal duration. The result showed that the algorithm is effective to recognize high-speed missile target.
\end{abstract}

KEYWORD: quasi-electrostatic field; high-speed target recognition; capacitance detector; algorithm

\section{INTRODUCTION}

It is well known that laser detector or infrared detector is common used in detection unit in active protection system[1]. With the development of antiarmor weapons, it need to react in the work area which is about $1 \mathrm{~m}$ away from target to achieve the goal of effective against anti-armor projectile which velocity is higher than $1000 \mathrm{~m} / \mathrm{s}$. This requires very short reaction time for detection. The laser or infrared system is difficult to quickly and accurately measure such motion parameters of high-speed projectile targets as distance and speed, while the capacitance detection with mature technology can do it well. In this way, the capacitance detection is a good choice for our research.

Based on the experiment for the static characteristics of target and background characteristics, we proposed high-speed target recognition algorithm, which feasibility is verified by the simulation.

\section{THEORY OF CAPACITANCE DETECTOR}

When applying alternating voltage to two induction electrodes of oscillation circuit of the capacitance detector, a detection physical field is established in a free space. Based on Electromagnetic theory, we can get:
$R_{\max }<<\frac{\lambda}{2 \pi}$

Where $R_{\max }$ is the maximum detection range in capacitance detection, $\lambda$ is the wavelength of capacitance detector. It shows that capacitance detection physical field is near-field area (Static area). In near-field area, the characteristic of field is mainly acted as electric field ${ }^{[2]}$. It knows that Quasistatic field within capacitance detector work area is mainly acted as quasi-electrostatic field.

This quasi-electrostatic field will be disturbed when target is near the capacitance detector and the capacitance amount between detector electrodes will be changed. The target detector can pick up the capacitance variety in the form of voltage in order to realize the target identification[3]. The function principle of capacitance detector is shown in figure 1. This detector is composed of regulator circuit, oscillation circuit, electrode voltage divider coupler and detector circuit. The variation of capacitance amount $\Delta C_{a d}$ between induction electrode $\mathrm{A}$ and ground electrode $\mathrm{D}$ will be generated in missiledetector encounter, the variation of oscillation circuit output voltage $\Delta U_{A}\left(\Delta U_{A}<0\right)$ is caused by $\Delta C_{a d}$. At the same time, impedance ratio $K r$ of electrode voltage divider coupler formed from detection electrodes A, B and D. $K r$ is defined as:

$$
K_{r}=\frac{c_{b d}+\Delta c_{b d}}{c_{a b}+\Delta c_{a b}}
$$


Where $C_{b d}$ is the capacitance between induction electrode $\mathrm{B}$ and ground electrode $D, C_{a b}$ is the mutual capacitance between induction electrode $\mathrm{A}$ and $\mathrm{B}, \Delta C_{b d}$ and $\Delta C a b$ are the variation.

$\Delta K r$ leads to the variation $\Delta K_{R}$ of Coupled divider ratio $K_{R}$, the relation of $K_{R}$ and $K r$ is as bellows:

$$
K_{R}=\frac{c_{a b}}{c_{a b}+c_{b d}}=\frac{1}{1+K_{r}}
$$

When a target encounters the capacitance detector, we can get:

$$
\begin{aligned}
& U_{i m}=K_{R} \bullet U_{A} \\
& U_{d}=K_{d d} \bullet U_{i m}+K_{s s}
\end{aligned}
$$

Here, $U_{i m}$ is the input voltage of detector circuit, $U_{d}$ is the output voltage of detector circuit, $K_{d d}$ and $\mathrm{K}_{\mathrm{ss}}$ are constants dependent on the structure of detector circuit. The variation and variation rate of $U_{d}$ constitute the target characteristics in missiledetector encounter[4].

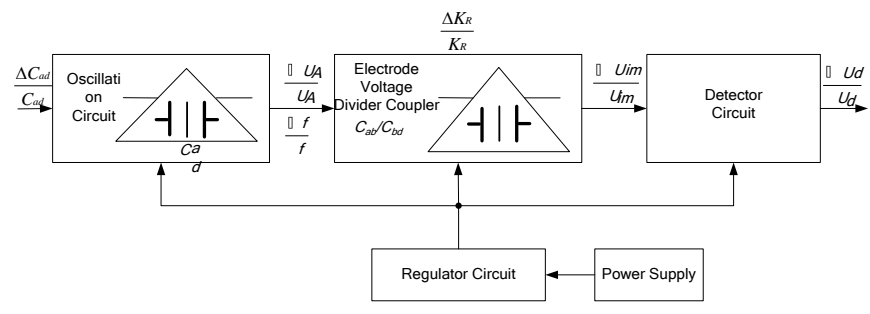

Fig.1 The schematic block diagram of capacitance detector.

\section{ALGORITHM IMPLEMENTATION}

The change of $U_{d}$ and $\Delta U_{d}$ can be used as recognition information for detector. In terms of the trend of detector circuit output voltage, target characteristics tested in laboratory conditions is in accord with detector encounters a real high-speed target. We do experiments to get the relationship between distance and detector circuit output voltage in missile-detector encounter with different

\begin{tabular}{|c|c|c|c|c|c|c|c|c|}
\hline \multirow{2}{*}{$H(\mathrm{~cm})$} & \multicolumn{2}{|c|}{$\begin{array}{c}\text { Encounter Center } \\
\text { of detector }\end{array}$} & \multicolumn{2}{|c|}{$\begin{array}{l}\text { Encounter Middle of } \\
\text { the left of detector }\end{array}$} & \multicolumn{2}{|c|}{$\begin{array}{l}\text { Encounter Top of } \\
\text { the left of detector }\end{array}$} & \multicolumn{2}{|c|}{$\begin{array}{l}\text { Encounter Below of } \\
\text { the left of detector }\end{array}$} \\
\hline & $U d(\mathrm{v})$ & $\Delta U d(\mathrm{mv})$ & $U d(\mathrm{v})$ & $\Delta U d(\mathrm{mv})$ & $U d(\mathrm{v})$ & $\Delta U d(\mathrm{mv})$ & $U d(\mathrm{v})$ & $\triangle U d(\mathrm{mv})$ \\
\hline 100 & 10.534 & -1 & 10.505 & 0 & 10.557 & -1 & 10.598 & 0 \\
\hline 90 & 10.533 & -2 & 10.504 & -1 & 10.555 & -3 & 10.597 & -1 \\
\hline 80 & 10.532 & -3 & 10.503 & -2 & 10.554 & -4 & 10.596 & -2 \\
\hline 75 & 10.53 & -5 & 10.502 & -3 & 10.553 & -5 & 10.594 & -4 \\
\hline 70 & 10.528 & -7 & 10.5 & -5 & 10.551 & -7 & 10.592 & -6 \\
\hline 65 & 10.526 & -9 & 10.497 & -8 & 10.549 & -9 & 10.589 & -9 \\
\hline 60 & 10.523 & -12.5 & 10.494 & -11 & 10.546 & -12.5 & 10.585 & -13 \\
\hline 50 & 10.51 & -25 & 10.484 & -21 & 10.54 & -18 & 10.579 & -19 \\
\hline 45 & 10.5 & -35.5 & 10.473 & -32 & 10.532 & -26 & 10.573 & -25 \\
\hline 40 & 10.483 & -52 & 10.461 & -44 & 10.522 & -36 & 10.564 & -34 \\
\hline 35 & 10.461 & -74.5 & 10.44 & -65 & 10.511 & -47 & 10.553 & -45 \\
\hline 30 & 10.415 & -120 & 10.397 & -108 & 10.491 & -67 & 10.534 & -64 \\
\hline 25 & 10.337 & -198 & 10.354 & -151 & 10.464 & -94 & 10.508 & -90 \\
\hline 20 & 10.192 & -343 & 10.269 & -236 & 10.428 & -130 & 10.475 & -123 \\
\hline 10 & 9.282 & -1253 & 9.945 & -560 & 10.339 & -219 & 10.4 & -198 \\
\hline
\end{tabular}
orientations, the data shows as tables 1 .

Table 1. The static characteristic data of approaching target in different orientations.

In table $1, O$ is the orientation in which target encounters the detector, $H$ is the vertical distance between the target and the capacitance detector, $U$ is the output voltage of detector circuit. Comparing data of the table, we can find out that output voltage $U_{d}$ of detector circuit decreases and the variable of $\Delta U_{d}$ increases when missile target gets close to the detector. In comparison with other three orientations, decreases trend of $U_{d}$ is faster when target encounters the center of detector. The decreases trend of $U_{d}$ is equally common between the situation of target encountering top of the left of detector and that below of the left of detector.

Based on the detector circuit output voltage, we use matlab curve fitting target Characteristics to make it more clearly. It shows obviously in figure2. 


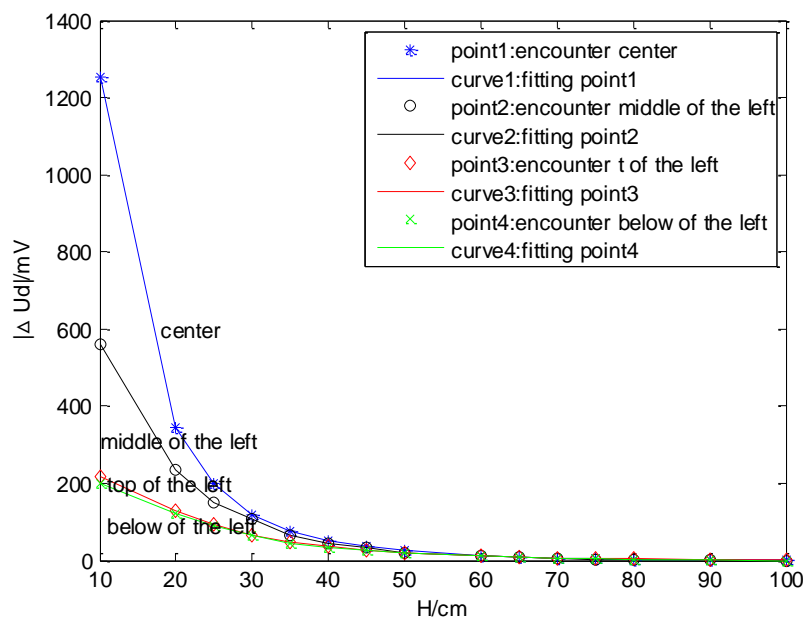

Figure 2. The static characteristics of target which approach detector in four orientations.

Here, $\left|\Delta U_{d}\right|$ is the absolute value of $\Delta U_{d}$. From analysis of capacitance detection theory, the detector circuit output voltage when electrodes and the target at a distance $100 \mathrm{~cm}$ measured as target far away from electrodes infinitely. In figure 3, with target coming near in the same orientation, time rate of change $\left|\Delta U_{d}\right|$ of the detector circuit output voltage gradually increase. The slope of $\left|\Delta U_{d}\right|$ increase little by little. In other words, second Derivative of detector circuit output voltage increase.

From analysis of the same target-missile encounter in different orientations, when target encounters the center of capacitance detector, values of $\left|\Delta U_{d}\right|$ and slope of $\left|\Delta U_{d}\right|$ are the maximum for the same distance. When target encounters top or below of the left of capacitance detector, values of $\left|\Delta U_{d}\right|$ and slope of $\left|\Delta U_{d}\right|$ are the minimum for the same distance. The measuring result is in good agreement with capacitance detection theory. And values of $\left|\Delta U_{d}\right|$ and slope of $\left|\Delta U_{d}\right|$ must be between the two limits when target encounters capacitance detector in other orientations. For this reason, target recognition signal is given if required distance between target and detector is from $15 \mathrm{~cm}$ to $25 \mathrm{~cm}$, values of $\left|\Delta U_{d}\right|$ and slope of $\left|\Delta U_{d}\right|$ can be regarded as discriminant criterion. Therefore, we can set change amount of $\left|\Delta U_{d}\right|$ within a certain time to be a threshold value. In the case of target encounters the center of detector and the distance is $25 \mathrm{~cm}$, threshold is met. And in the case of target encounters the top or below of the left of detector and the distance is $15 \mathrm{~cm}$, threshold should also be met. According to structure symmetry of capacitor detector, threshold will be met when target encounters detector in other orientations and distance between them is from $15 \mathrm{~cm}$ to $25 \mathrm{~cm}$.

In order to eliminate the affection of interference signal, we set target duration and the initial value of the change amount of detector circuit output voltage to be a threshold value. Common interfering objects such as tree branches, human, vehicles, small bullets. Compared with $1000 \mathrm{~m} / \mathrm{s}$ high speed targets, branches, the human body and the vehicle belong to the larger objects, the speed of closing to the detector is very low. Detector circuit output voltage amplitude caused by the larger objects changes greatly, but values of $\left|\Delta U_{d}\right|$ and slope of $\left|\Delta U_{d}\right|$ are small. In this case, we can set the continuous change amount of $\left|\Delta U_{d}\right|$ within a certain time to identify target and larger interfering objects at low speed. Comparing of $1000 \mathrm{~m} / \mathrm{s}$ high-speed target and small bullet, we can find out that the change rate of detector circuit output voltage caused by small bullet is relatively close to that of high-speed missile target. However, the bullet is small in size, the change amount of detector circuit output voltage is small when small bullet is coming near. In this case, we can set initial value of detector circuit output voltage within a predetermined distance range to be a threshold value, which is used to identify target and small interfering objects at high speed.

For these reasons, we can get high-speed target identification criterion. With the missile target coming near, detector circuit output voltage $U_{d}$ decreases, the change amount and the second derivative of $U_{d}$ increase. Within a predetermined distance range from $15 \mathrm{~cm}$ to $25 \mathrm{~cm}$, the change amount of $U_{d}$ should be greater than threshold value A. The continuous change amount of $\left|\Delta U_{d}\right|$ within a certain time should be greater than threshold value B. For the setting of threshold value A, in figure 2, we can get the change amount $\left|\Delta U_{l}\right|$ of detector circuit output voltage when missile target encounters the top or below of the left of detector and the distance is $15 \mathrm{~cm}$. And we can get the change amount $\left|\Delta U_{c}\right|$ of detector circuit output voltage when target encounters the center of detector and the distance is $25 \mathrm{~cm} .\left|\Delta U_{l}\right|$ is relatively close to $\left|\Delta U_{c}\right|$. We can set $\left|\Delta U_{l}\right|$ to be threshold value $\mathrm{A}$. Threshold value $\mathrm{A}$ will be met when high-speed missile target encounters detector in other orientations and distance range between them is from $15 \mathrm{~cm}$ to 25 $\mathrm{cm}$. Considering the interfering signal and the possibility with a low probability of occurrence that missile target encounters top or below of the left of capacitance detector, threshold value $A$ can be greater than $\left|\Delta U_{1}\right|$ appropriately. For the setting of threshold value $\mathrm{B}$, when missile target encounters the detector at speed of $1000 \mathrm{~m} / \mathrm{s}$, Target motion distance is $3 \mathrm{~cm}$ for $30 \mathrm{~s}$. When the distance range between missile target and detector is from $15 \mathrm{~cm}$ to $25 \mathrm{~cm}$, minimum value of change amount of $\mid \Delta U_{d}$ | for $3 \mathrm{~cm}$ is set to be threshold value B. In figure 2, when missile target encounters the top or below of the left of detector and the range between them is from $15 \mathrm{~cm}$ to $18 \mathrm{~cm}$, we can get the minimum value $60 \mathrm{mV}$ of continuous change amount of $\left|\Delta U_{d}\right|$, which is set to be threshold value $\mathrm{B}$. Considering the targets speed may be less than $1000 \mathrm{~m} / \mathrm{s}$, threshold value B can be less than $60 \mathrm{mV}$ appropriately.

For the sake of recognition high-speed missile target accurately, the signal conditioning circuit will 
give a signal $K\left|\Delta U_{d}\right|(K>1)$ by a reverse amplification process of $\Delta U_{d} . K\left|\Delta U_{d}\right|(K>1)$ is the input of target recognition algorithm. The algorithm implementation is shown as below:

1) Take $t_{w} \mu \mathrm{s}$ as the duration of input data. Assumed that $\mathrm{M}$ data points have been detected before $t \mu \mathrm{s}\left(t>t_{w}\right)$, then we can get $(\mathrm{M}+1)$ data points: $x(t), x\left(t-t_{s}\right), x\left(t-2 t_{s}\right), \ldots, x\left[t-(\mathrm{M}-1) t_{s}\right]$, $x\left(t-\mathrm{M} t_{s}\right)$. Here, $f_{s}$ is the sampling frequency of $K$ $\left|\Delta U_{d}\right|, \mathbf{M}=t_{w} \times f_{s}, t_{s}=1 / f_{s}$.

2) Data acquisition is implemented every $t_{s} \mu \mathrm{s}$. We can get $(\mathrm{M}+1)$ data points: $x\left(t+j \times t_{s}\right), x[t+(j$ $\left.-1) \times t_{s}\right], x\left[t+(j-2) \times t_{s}\right], \ldots, x\left[t+(j-\mathrm{M}+1) \times t_{s}\right]$, $x\left[t+(j-\mathbf{M}) \times t_{s}\right]$. Here, the value of $j$ is $0,1,2,3 \ldots$

$3)$ If the accessed $(M+1)$ data points in serial meet all the criterion shown as below: $x\left(t+j \times t_{s}\right)>$ $x\left[t+(j-1) \times t_{s}\right]>x\left[t+(j-2) \times t_{s}\right]>\ldots>x[t+(j-$ $\left.\mathrm{M}+1) \times t_{s}\right]>x\left[t+(j-\mathrm{M}) \times t_{s}\right],\left\{x\left(t+j \times t_{s}\right)-x[t+\right.$ $\left.\left.(j-1) \times t_{s}\right]\right\}>\left\{x\left[t+(j-1) \times t_{s}\right]-x[t+(j-2) \times\right.$ $\left.\left.t_{s}\right]\right\}>\ldots>\left\{x\left[t+(j-\mathrm{M}+1) \times t_{s}\right]-x[t+(j-\mathrm{M}) \times\right.$ $\left.\left.t_{s}\right]\right\}, x\left[t+(j-\mathrm{M}) \times t_{s}\right]>$ threshold value $\mathrm{A}$, and $\{x(t$ $\left.\left.+j \times t_{s}\right)-x\left[t+(j-\mathrm{M}) \times t_{s}\right]\right\}>$ threshold value $\mathrm{B}$, then we can verify the accessed $(M+1)$ data points as target signal and recognize high-speed missile target.

\section{SIMULATION AND VALIDATION}

In order to verify the validity of the high-speed target recognition algorithm, the validation of simulation model based on target recognition process is presented, which is in ISE13.3 environment using the Isim software.

The simulation input is binary code stream ad_data[11:0] converted by data $K\left|\Delta U_{d}\right|$, which is actually measured when the approximately $1000 \mathrm{~m} / \mathrm{s}$ high-speed missile target encounters detector. Discrete values of ad_data[11:0] range from 0 to 4095. Each discrete value is represented with 12 bits binary. If input data beyond range of ad_data[11:0], overflow flag is output. The simulation wave is shown as figure 3 .

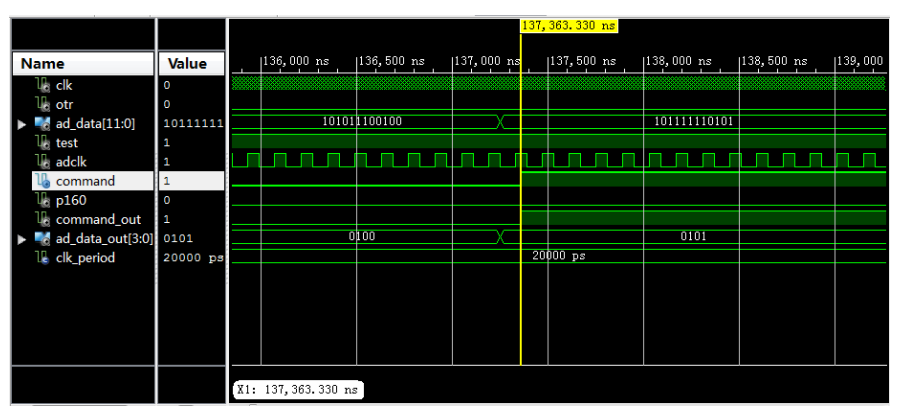

Figure 2. The simulation wave after inputting target signals.
In figure 3 , after setting of $50 \mathrm{MHz}$ clock signal clk, overflow flag otr and test signal test, we enter high-speed data input target signal ad_data[11:0] into Isim software. Recognition signal command jump to high level 1 from low level 0 according to the algorithm proposed in this paper. In other words, the system recognizes high-speed missile target at the moment of $137.36 \mu \mathrm{s}$. At this moment, value of input data ad_data[11:0] is 10111110101, value of $K$ $\left|\Delta U_{d}\right|$ is $3.7 \mathrm{~V}$, and the distance between missile target and the center of detector is $16 \mathrm{~cm}$. This distance is within the recognition range of $15 \mathrm{~cm}$ to $20 \mathrm{~cm}$, which in accordance with the static measurement distance.

\section{CONCLUSION}

Based on capacitance detection theory and the static test experiment under laboratory conditions for simulating actual missile-detector encounter, this paper analyzed characteristics of high-speed missile target, set the recognition threshold, determined target recognition algorithm and verified the correctness and validity of the algorithm using the measured data. Advantages of method proposed in this paper are as followings: this method takes full advantage of characteristics of precise ranging of capacitance detector; threshold value set in this paper can recognize high-speed missile targets which encounter the detector in different orientations; this paper verifies the algorithm by measured data simulation, which reduces the experiment cost and improves the experimental accuracy. What's more, this algorithm has extensive applicability. Through further optimization, this algorithm can not only recognize the same high-speed missile target which encounters detector in different orientations, but also provide the useful reference to precise recognition in the case of different high-speed missile targets encountering the same detector.

\section{REFERENCES}

[1] REN Xiaogang. 2010. Foreign Tank Armored Vehicle Active Protection System Introduction. Fire Control and Command Control 35: 4-6.

[2] DENG Jiahao, ZHAN Yi, SHI Jusheng. 2000. The theoretical Analysis on the Field Zone of a Capacitance Detector. Journal of Detection \& Control 22(2): 14-16.

[3] Cui Ping, Qi Xinlin, Yu Chunhua, Yang Yanfeng. 2012. Design of The Electric Properties Test System of Capacitance Proximity Fuze for HE. Proceedings of 2012 International Conference on Quality, Reliability, Risk, Maintenance, and Safety Engineering.

[4] Lu Shilong. 2013. Target detecting technology of Active Protection System based on Static Electric Field. 2013 IEEE International Conference on Computer Science and Automation Engineering. 